\title{
BIBLIOGRAPHY
}

1. J. Lillo, Continuous matrices and the stability theory of differential systems, Math. Z. vol. 73 (1960) pp. 45-58. 138.

2. —_, Perturbations of nonlinear systems, Acta Math. vol. 103 (1960) pp. 123-

3. - Linear differential equations with almost periodic coefficients, Amer. J. Math. vol. 81 (1959) pp. 37-45.

4. L. Markus, Continuous matrices and the stability of differential systems, Math. Z. vol. 62 (1955) pp. 310-319.

5. W. T. Reid, Remarks on a matrix transformation for linear differential equations, Proc. Amer. Math. Soc. vol. 8 (1957) pp. 708-712.

RIAS

\section{METRIC TERNARY DISTRIBUTIVE SEMI-LATTICES}

\author{
S. P. AVANN
}

In this paper we show that the ternary operation of a metric ternary distributive semi-lattice, a generalization of the ternary Boolean algebra of Grau [2], uniquely minimizes ternary distance. This generalizes a result of Birkhoff and Kiss [1, Corollary 1, p. 749]. We show, conversely, that in a metric space unique minimizing of ternary distance determines a ternary operation with respect to which the space is a ternary distributive semi-lattice. Particularly, a lattice whose graph satisfies the unique minimal ternary distance condition and certain finiteness conditions must be distributive. This answers a question proposed by Birkhoff and Kiss [1, p. 750].

1. Definitions and postulates. We state our results at the close of this section.

A ternary distributive semi-lattice, hereinafter abbreviated TDSL, is a set of $J$ elements closed with respect to a ternary operation $(a, b, c)$ satisfying the following identities.

(T1) $(a, a, b)=a$.

(T2) $(a, b, c)$ is invariant under all 6 permutations.

(T3) $(a,(b, c, d), e)=((a, b, e), c,(a, d, e))$.

REMARK. The term, introduced by the author (Abstract 86, Bull. Amer. Math. Soc. vol. 54 (1948) p. 79), is a natural one in view of Lemma 3. If in Lemma 3 there exists $a^{\prime} \in J$ satisfying

Received by the editors April 11, 1960 and, in revised form, July 18, 1960. 
(T4) $\left(a, b, a^{\prime}\right)=b$ for all $b \in J$

then $\mathcal{P}(a, J)$ is a distributive lattice with $a$ and $a^{\prime}$ as zero and unit elements. If also $\mathfrak{I}$ satisfies:

(T5) For each $a \in J$ there exists a complement $a^{\prime} \in J$ satisfying (T4), then $J$ becomes the Ternary Boolean Algebra of Grau [2] and $\mathcal{P}(a, T)$ is a Boolean Algebra for each $a \in J$.

By a suitable permutation of the letters in (T3) Sholander in [4, p. 801 ] was able to replace (T2) and (T3) by a single postulate (N). $\mathrm{His}(\mathrm{M})$ is (T1).

We remark here that by virtue of (T2), (T3) can be written and applied with many variations; particularly, the solo element in the right member can be $b$ or $d$.

In a metric space $\mathfrak{T}$ we denote distance by $b c$ and introduce ternary distance $[x ; b, c, d]=x b+x c+x d$.

We shall be concerned with an undirected graph $\mathcal{g}$ with no loops, i.e., the graph of a symmetric anti-reflexive binary relation $R$ on a set of elements: $a R a$ is false for all $a \in \mathcal{J}$ and $a R b$ iff $b R a$. Two elements $b$ and $c$ are vertices of an edge iff $b R c$. Moreover, when $g$ is connected, it is a metric space with respect to distance defined: $b b=0 ; b c=1$ iff $b R c ; b c=n$ iff $b R b_{1} R \cdots R b_{n}=c$ is a minimal such sequence. An even graph is one with no odd-sided polygons $b_{1} R b_{2} R \cdots R b_{2 n+1} R b_{1}$.

The graph $g(P)$ of a partially ordered set $P$ is defined by: $b R c$ iff $b<c$ or $b>c$ ( $<$ : is covered by).

We shall deal with the following two minimal ternary distance postulates in a metric space $\mathfrak{T}$ and a corresponding ternary operation for each.

(U) For each (unordered) triple $b, c, d \in \mathscr{N}$ there exists a unique $t \equiv[b, c, d] \in \mathbb{N}$ such that $[t ; b, c, d]=(b c+c d+d b) / 2$.

(V) For each triple $b, c, d \in \mathfrak{N}$ there exists a unique $s \equiv[b, c, d] \in \mathfrak{N}$ such that $[s ; b, c, d]<[x ; b, c, d]$ for all $x \in \mathscr{N}, x \neq s$.

By virtue of Lemma 1 we shall see that (U) implies (V) in $\mathfrak{T}$.

Ternary betweenness relations and notation are defined as follows:

(TB) In a TDSL $J,(b x c) \leftrightarrow(b, x, c)=x$.

(MB) In a metric space $\mathfrak{T}, b x c \leftrightarrow b x+x c=b c$.

(VB) In a graph $g$ satisfying (V) or (U), $[b x c] \leftrightarrow[b, x, c]=x$.

Finiteness conditions in terms of convex sets are defined as follows:

(TF) In a TDSL $J,\{x \in J \mid(b x c)\}$ is finite for all $b, c \in J$.

(MF) In a metric space $\mathfrak{N},\{x \in \mathfrak{N} \mid b x c\}$ is finite for all $b, c \in \mathfrak{N}$.

(VF) In a graph $\mathcal{g}$ satisfying (V) or (U), $\{x \in \mathfrak{g} \mid[b x c]\}$ is finite for all $b, c \in \mathcal{J}$.

When (TF) holds we define the graph $g(J)$ of a TDSL $J$ as follows: $b R c$ iff $b \neq c$ and $(b, x, c)=b$ or $c$ for all $x \in \mathfrak{J}$. $g(J)$ will be connected, 
as shown in Lemmas 4 and 7, and therefore metrizable in the manner described above.

We now summarize our results.

THEOREM 1. If $\mathrm{J}$ is simultaneously a TDSL and a metric space in which (TB) and (MB) are equivalent: $(b x c) \leftrightarrow b x c$, then (U) is satisfied (and also (V)).

TheOREM 2. If a TDSL J satisfies (TF), then the metric space $\mathrm{J}(\mathfrak{J})$, as defined and metrized above, satisfies (U). Moreover (TB) and (MB) are equivalent.

THEOREM 3. A metric space $\mathfrak{T}$ satisfying (U) is a TDSL with respect to the ternary operation $[b, c, d]$. Moreover (MB) is equivalent to (VB) (which is (TB)).

We define a unique ternary distance graph $\mathcal{g}$, hereinafter called a UTD graph, as one satisfying (MF) and (V).

TheOREM 4. A UTD graph satisfies (U) and is a TDSL with respect to the ternary operation $[b, c, d]$. Moreover (MB) and (VB) are equivalent.

THEOREM 5. If every $a \in \mathfrak{L}$, a lattice with zero element $z$, is of finite dimension, and if the graph $\mathcal{g}(\mathcal{L})$ satisfies (MF) and (V), then $\mathcal{L}$ is distributive.

2. Ternary distributive semi-lattices. In this section we consider a TDSL which is a metric space and prove Theorems 1 and 2.

LEMMA 1. In any metric space $\mathfrak{T}$

(MT1) $[x ; b, c, d] \geqq(b c+c d+d b) / 2$,

(MT2) $[x ; b, c, d]=(b c+c d+d b) / 2 \leftrightarrow b x c \cdot c x d \cdot d x b$.

Proof. (MT1) follows from taking one-half the sum of the inequalities $b x+x c \geqq b c, c x+x d \geqq c d, d x+x b \geqq d b$. Clearly equality holds simultaneously in all three iff equality holds in (MT1).

LEMMA 2. In a TDSL $\boldsymbol{J}(b t c) \cdot(c t d) \cdot(d t b)$ is satisfied uniquely by $t=(b, c, d)$, where $\cdot$ denotes logical conjunction.

This follows easily from (T1-2-3). See [3, 8.4 and 8.13].

Proof of Theorem 1. Since (TB) $\leftrightarrow(\mathrm{MB})$, by Lemma 2 we have $b t c \cdot c t d \cdot d t b$ holding uniquely for $t=(b, c, d)$. Whence by (MT2) and (MT1) resp. $[t ; b, c, d]=(b c+c d+d b) / 2<[x ; b, c, d]$ for all $x \neq t$.

LEMMA 3. For each $a \in J, a T D S L$, the elements of $\mathfrak{J}$ constitute a distributive semi-lattice $\mathcal{P}(a, J)$, closed with respect to symmetric join of 
meets of triples (called by Sholander a median semi-lattice) as follows:

(1) The inclusion relation is given by $b \subseteq_{a} c\left(\right.$ and $\left.c \supseteq_{a} b\right) \leftrightarrow(a, b, c)=b$.

(2) The zero element is a.

(3) $\mathcal{P}(a, J)$ is closed with respect to meet given by $b \cap_{a} c=(b, a, c)$.

(4) Existence of common upper bound $b \subseteq_{a} m$ and $c \subseteq_{a} m$, implies the join exists and is given by $b \cup_{a} c=(b, m, c)$.

(5) Distributivity: existence of $b \cup_{a} c$ implies $d \cap_{a}\left(b \cup_{a} c\right)=\left(d \cap_{a} b\right)$ $\cup_{a}\left(d \cap_{a} c\right)$.

(6) For all triples $b, c, d$ there exists $\left(b \cap_{a} c\right) \cup_{a}\left(c \cap_{a} d\right) \cup_{a}\left(d \cap_{a} b\right)$, which is $(b, c, d)$.

The proof is a routine application of the postulates and is done in [5, pp. 809-810].

Lemma 4. Every principal ideal of $\mathcal{P}(a, J)$, namely $\mathcal{P}(a, m)$ $=\{x \mid(a x m)\}$, is a distributive lattice, which is finite if (TF) is satisfied.

Proof. The lemma follows from (4) of Lemma 3 and the fact that one distributive law implies the other.

Lemma 5. In a TDSL J $(a b c) \cdot(a c d) \leftrightarrow(a b d) \cdot(b c d)$.

We prove this known result to illustrate applications of the postulates. If $(a b c) \cdot(a c d)$, then $(a, b, d)=(a,(a, b, c), d)=((a, a, d), b,(a, c, d))$ $=(a, b, c)=b$ yielding $(a b d)$. Also $(b, c, d)=((a, b, c), c, d)$ $=((a, c, d), b,(c, c, d))=(c, b, c)=c$ so that $(b c d)$ subsists. The converse holds by symmetry.

LeMma 6. In $\odot(a, \mathfrak{J}), b$ is covered $b y c \neq b: b<{ }_{a} c\left(c>{ }_{a} b\right)$ iff $(a, b, c)=b$ and $(b, x, c)=b$ or $c$ for all $x \in J$.

Proof. Let $b<{ }_{a} c$. Then $(b, a, c)=(a, b, c)=b$ and $(a b c)$. For arbitrary $x \in J$ let $(b, x, c)=d$. Then also $(b d c)$ by Lemma 2 . Applying Lemma 5 with roles of $c$ and $d$ interchanged, we obtain $(a b d) \cdot(a d c)$. By Lemma $3 a \subseteq_{a} b \subseteq_{a} d \subseteq_{a} c$, and the hypothesis requires $d=b$ or $d=c$. Conversely, let $(b, x, c)=b$ or $c$ for all $x$ and $(b, a, c)=b$. Then immediately $b \subseteq_{a} c$. Assume $a \subseteq_{a} b \subseteq_{a} x \subseteq_{a} c$ so that $(a b x) \cdot(a x c) \cdot(a b c)$. By Lemma $5(b x c)$ so that $x=(b, x, c)$, which must be $b$ or $c$ as desired.

Lemma 7. In a TDSL $\mathfrak{J}$ satisfying (TF), $b R c$ in $\mathcal{J}(\mathfrak{J})$ iff $b R_{a} c$ in $\mathcal{J}(\mathcal{P}(a, J))$, where $R_{a}$ is $\lessgtr_{a}$. Thus $\mathcal{J}(\mathcal{J})$ and $\mathcal{J}(\mathcal{P}(a, J))$ are isometric.

Proof. In $g(J) b R c$ iff $(b, x, c)=b$ or $c$ for all $x$ including $(b, a, c)=b$ or $c$. Hence by Lemma $6 b R c$ iff $b \lessgtr_{a} c$ in $\odot(a, J)$ iff $b R_{a} c$ in $g(\odot(a, J))$.

LEMMA 8. In a TDSL I satisfying (TF), (TB) and (MB) are equivalent. 
Proof. Given $(a b c)$. Then $(a, b, c)=b$ and $b \subseteq_{a} c$ in the principal ideal $P(a, c)$. The latter is a finite distributive lattice by Lemma 4 and satisfies the Jordan-Dedekind chain condition. Therefore a chain $a<{ }_{a} a_{1}<_{a} \cdots<_{a} a_{m}=b \in \mathcal{P}(a, c)$ exists and minimizes a sequence $a R_{a} x_{1} R_{a} \cdots R_{a} b$, where $R_{a}$ is $\lessgtr_{a}$. The corresponding sequence of $g(J)$ of Lemma 7: $a R a_{1} R \cdots R a_{m}=b$ is thus minimal so that $a b$ $=\delta_{a}[b]$, the dimension of $b$ in $\odot(a, \mathfrak{J})$. Similarly $a c=\delta_{a}[c]$. Again, $b<_{a} b_{1}<_{a} \cdots<_{a} b_{n}=c$ minimizes sequences $b R_{a} \cdots R_{a} c$ and by virtue of Lemma 7 yields a corresponding minimal sequence $b R b_{1} R$ $\cdots R b_{n}=c$ of $\mathcal{J}(J)$ of length $b c=n$. The total chain $a<_{a} a_{1}<_{a} \cdots$ $<_{a} a_{m}=b<_{a} b_{1}<_{a} \cdots<_{a} b_{n}=c$, again in view of the Jordan-Dedekind chain condition in $\odot(a, c)$, yields a minimal chain $a R a_{1} R \cdots R a_{m}$ $=b R b_{1} R \cdots R b_{n}=c$. Hence $a c=\delta_{a}[c]=\delta_{a}[b]+b c=a b+b c$, yielding $a b c$. Conversely suppose $a b c$ holds. Let $d=(a, b, c)$. By Lemma 2 $(a d b) \cdot(b d c) \cdot(c d a)$. By the proof just completed $a d b \cdot b d c \cdot c d a$. Hence $0=(a d+d b-a b) / 2+(b d+d c-b c) / 2-(c d+d a-c a) / 2$ $=b d-(a b+b c-c a) / 2=b d-(a c-c a) / 2=b d$. Thus $b=d,(a, b, c)=b$, and $(a b c)$.

Proof of Theorem 2. Lemma 8 completes the hypothesis of Theorem 1.

3. Unique ternary distance graphs. We prove Theorems 3,4 and 5 in this section.

LEMMA 9. In a metric space satisfying (U), (VB) and (MB) are equivalent.

Proof. [bcd] iff $c=[b, c, d]$ iff $[c ; b, c, d]=b c+c c+c d$ $=(b c+c d+d b) / 2$ iff $b c+c d=b d$ iff $b c d$.

Lemma 10. (Condition (D) of Sholander [4, p. 804]). For each unordered triple $b, c, d \in \mathfrak{N}$, a metric space satisfying (U), there exists a unique $s \in \mathbb{N}$ such that $b s c \cdot c s d \cdot d s b$, namely $s=[b, c, d]$.

Proof. By (U) there exists unique $s=[b, c, d]$ such that $[s ; b, c, d]$ $=(b c+c d+d b) / 2$. We apply Lemma 1 . By (MT2) $b s c \cdot c s d \cdot d s b$, and for $x \neq s[x ; b, c, d]>(b c+c d+d b) / 2$ so that at least one of $b x c, c x d$, $d x b$ fails.

LEMMA 11. In any metric space $a b c \cdot a c d \leftrightarrow a b d \cdot b c d$.

This is an elementary property of metric spaces.

Proof of Theorem 3. The metric betweenness relation $b c d$ satisfies the set of conditions $\Sigma_{1}\left(D, B_{1}, F\right)$ of Sholander [4, pp. 803-805]: (D) by Lemma 10 ; $\left(\mathrm{B}_{1}\right) a b a \rightarrow a=b$, trivially; and (F) $a b c \cdot a c d$ 
$\rightarrow d b a(\leftrightarrow a b d)$ by Lemma 11 . By Lemma 9 the equivalent betweenness relation $[b c d]$ also satisfies $\Sigma_{1}$. Sholander showed in $[4,4.10]$ that the corresponding ternary operation $[b, c, d]$ satisfies his conditions $(\mathrm{M})$ and $(\mathrm{N})$. The latter, he showed in $[3,8.3]$, are equivalent to (T1-2-3).

Corollary to TheORem 3. If a metric space $\mathfrak{T}$ satisfies (U) and for some pair $a, a^{\prime} \in \mathfrak{T} a x a^{\prime}$ for all $x \in \mathfrak{N}$, then $\odot(a, \mathfrak{J})=\Phi\left(a, a^{\prime}\right)$ is $a$ distributive lattice with $a$ and $a^{\prime}$ as zero and unit elements.

Proof. By Theorem $3 \mathfrak{N}$ becomes a TDSL $J$ under $[b, c, d]$ with [axa'] for all $x \in$ J. I.e., $a \subseteq_{a} x \subseteq_{a} a^{\prime}$ for all $x$. Lemma 4 completes the proof.

LEMma 12. A necessary and sufficient condition that a connected graph d be even is that bRc implies $b x-c x= \pm 1$. Furthermore, a UTD graph is even.

Proof. Given $\mathcal{J}$ is even and suppose $b R c$. Then $1=b c \geqq b x-c x$ $\geqq-b c=-1$. But $b x \neq c x$ since $b x-c x+1 \equiv b x+c x+b c \equiv 0(\bmod 2)$. Hence $b x-c x= \pm 1$. Conversely suppose $d$ is not even. Two adjacent vertices $b, c$ and the opposite vertex $x$ of a smallest odd-sided polygon give $b c=1$ and $b x=c x$. Moreover $[c ; x, b, c]=c x+1=b x+1=[b ; x, b, c]$ $\leqq x y+b y+c y=[y ; x, b, c]$ for $b \neq y \neq c$. Hence $b$ and $c$ (and possibly $y$ also) are tied for minimal ternary distance from $x, b, c$ so that $g$ is not a UTD graph. We have thus proved the contrapositives of the converse and the second statement.

We may note at this point that in a UTD graph the ternary operation $[b, c, d]$ satisfies (T2) trivially by symmetry. It also satisfies (T1). For if $x \neq a,[x ; a, a, b]=a x+(a x+b x)>a a+a a+a b=[a ; a, a, b]$ so that $a=[a, a, b]$. We shall circumvent a direct proof of (T3), which would be tedious.

\section{LEMMA 13. In a UTD graph (MB) and (VB) are equivalent.}

Proof. First suppose $a b c$. If $x \neq b,[x ; a, b, c]=(a x+c x)+b x>a c$ $=a b+b c=[b ; a, b, c]$. Hence $b=[a, b, c]$ and $[a b c]$ subsists. Conversely, suppose $[a b c]$. We shall prove by induction on $n=b c$ that $a b c$ follows. We note that $a b c$ holds trivially for $n=0$. When $n=1$, $a b=a c \pm 1$ by Lemma 12. But $a b=a c+1=a c+b c$ yields $a c b$ and $[a c b]$ by the first part of this proof and leads to the contradiction $[a, b, c]$ $=[a, c, b]=c \neq b=[a, b, c]$. Thus $a c=a b+b c$ as desired. Assume $[a, b, c]=b$ implies $a b c$ whenever $n \leqq k$. Consider $[a, b, c]=b$ with $n=b c=k+1$. Let $b R b_{0}$ with $b_{0}$ on minimal $b-c$ chain: $b b_{0}=1$ and $b b_{0}+b_{0} c=b c$. Since $b=[a, b, c], a b+b c=[b ; a, b, c]<\left[b_{0} ; a, b, c\right]$ 
$=a b_{0}+\left(b b_{0}+b_{0} c\right)=a b_{0}+b c$. Thus $a b<a b_{0}$ and $a b_{0}=a b+1$ by Lemma 12. Now $\left[b ; a, b_{0}, c\right]=a b+1+b c>a b+b c=a b+1+b_{0} c=a b_{0}+b_{0} c$ $=\left[b_{0} ; a, b_{0}, c\right]$. Also for $x \neq b$ we apply hypothesis and Lemma 12 to obtain $\left[x ; a, b_{0}, c\right]=[x ; a, b, c]+\left(b_{0} x-b x\right) \geqq 1+[b ; a, b, c]+( \pm 1)$ $\geqq[b ; a, b, c]=a b+b c=a b_{0}+b_{0} c=\left[b_{0} ; a, b_{0}, c\right]$. Uniqueness of minimality in (V) requires that $b_{0}=\left[a, b_{0}, c\right]$ or $\left[a b_{0} c\right]$. But $b_{0} c=b c-1=k$. By the induction hypothesis $a b_{0} c$ subsists. Hence $a c=a b_{0}+b_{0} c$ $=a b+b c$ yielding $a b c$. The induction is complete.

Proof of Theorem 4. Let $s=[b, c, d]$. Then for $x \neq s[s ; b, s, c]$ $=(b s+c s+d s)-d s<b x+c x+(d x-d s) \leqq b x+c x+s x=[x ; b, s, c]$. Thus $s=[b, s, c]$ and $[b s c]$ subsists. Similarly $[c s d]$ and $[d s b]$. Then $b s c \cdot c s d \cdot d s b$ by Lemma 13 . By Lemma 1 and (V) we have $[s ; b, c, d]$ $=(b c+c d+d b) / 2<[x ; b, c, d]$ for all $x \neq s$. This is (U). Hence Theorem 4 now follows from Theorem 3 .

LEMma 14. In any lattice or semi-lattice if $a R b R c R d R a$, where $R$ is $\lessgtr$, alternate $R$ 's are opposite directional covering.

This follows by definition of covering and uniqueness of join and meet when they exist.

LEMMA 15. In a lattice $\mathfrak{L}$, for which $\mathrm{g}(\mathcal{L})$ is a UTD graph with respect to the ternary operation determined by the metric, $b \lessgtr c$ in $\&$ iff $b \neq c$ and $[b, x, c]=b$ or $c$ for all $x \in \mathcal{J}(\mathcal{L})$.

Proof. If $b \lessgtr c$ in $\&$, then by Lemma $12 \mathcal{g}(\mathfrak{L})$ is even and $b x-c x$ $= \pm 1= \pm b c$ in $g(\&)$. Thus $[b c x]$ or $[c b x]$, i.e., $[b, x, c]=c$ or $b$. If $b \$ c$, then $b=c$ or there exists $r \in \mathcal{J}(\mathcal{L})$ with $b, r, c$ all distinct such that $[b r c]$ or $[b, r, c]=r \neq b$ or $c$.

Proof of Theorem 5. Finite dimensionality of the elements of $\mathscr{L}$ makes $\mathcal{J}(\mathscr{L})$ well defined and connected through $z$, so that it is a UTD graph. Hence by Theorems 3 and $4 g(\mathscr{L})$ is a TDSL with respect to the operation $[a, b, c]$, and (MB) is equivalent to (VB) (which is (TB)). Moreover all the lemmas are valid and applicable. By Lemma 3 $P(z, g(\mathscr{L}))$ is a distributive semi-lattice with the same zero element $z$ of $\mathscr{L}$. We shall show that $\mathcal{P}(z, \mathcal{g}(\mathcal{L}))$ is isomorphic to $\mathscr{L}$ under the identity correspondence $c \leftrightarrow c$. Combining the results of Lemmas 6 and $15 b \lessgtr_{z} c$ in $\mathcal{P}(z, \mathcal{J}(\mathscr{L}))$ iff $b \lessgtr c$ in $\mathscr{L}$. Accordingly, it will be sufficient to show that

(S) $b<{ }_{z} c$ in $\mathcal{P}(z, \mathcal{J}(\mathscr{L}))$ implies $b<c$ in $\mathscr{L}$.

We employ an induction on $n=z c$, the distance from $z$ to $c$ in $\mathcal{g}(\mathscr{L})$, and note (S) is trivially true for $n=1: z<{ }_{z} c$ in $P(z, \mathcal{J}(\mathscr{L}))$ iff $z<c$ in $\mathscr{L}$. Assume (S) holds for $n \leqq k$. Now consider $b<{ }_{2} c$ with $z b=k$ and $z c$ $=k+1$ in $\mathscr{J}(\mathfrak{L})$, and assume that $b>c$ in $\mathscr{L}$. From all necessarily 
finite descending chains $b>c>\cdots$ in $\mathscr{L}$ select one with an earliest agreement of $>$ and $>_{2}: b=c_{0}<{ }_{2} c=c_{1}<{ }_{2} \cdots<{ }_{2} c_{r-1}<{ }_{2} c_{r}>{ }_{2} c_{r+1}$ $\lessgtr_{z} \ldots$. By Lemma 4 the ideal $P\left(z, c_{r}\right)$ of $P(z, \mathcal{J}(\mathcal{L}))$ is a distributive lattice. Hence by lower semi-modularity $c_{r-1}>{ }_{2} d=c_{r-1} \cap{ }_{2} c_{r+1}<{ }_{2} c_{r+1}$. On the other hand $c_{r-1}>c_{r}>c_{r+1}<d<c_{r-1}$, where the direction of the last two coverings are required by Lemma 14. If $r=1$, the induction hypothesis requires $b>d<c_{2}$ contradicting $c_{2}<d<b$. If $r>1$, then $c_{r-1}>d$ contradicts the minimality of $r$. Hence our assumption $b>c$ is false, and the induction on $n=z c$ for validity of $(\mathrm{S})$ is complete. Therefore $\mathscr{P}(z, \mathfrak{g}(\mathscr{L})$ ) is isomorphic to $\mathscr{L}$ and is a distributive lattice (rather than a semi-lattice). Thus $\mathscr{L}$ itself is distributive.

\section{REFERENCES}

1. G. Birkhoff and S. A. Kiss, A ternary operation in distributive lattices, Bull. Amer. Math. Soc. vol. 53 (1947) pp. 749-752.

2. A. A. Grau, Ternary Boolean algebra, Bull. Amer. Math. Soc. vol. 53 (1947) pp. 567-572.

3. Marlow Sholander, Trees, lattices, order, and betweenness, Proc. Amer. Math. Soc. vol. 3 (1952) pp. 369-381.

4. - Medians and betweenness, Proc. Amer. Math. Soc. vol. 5 (1954) pp. 801-807.

5. - Medians, lattices, and trees, Proc. Amer. Math. Soc. vol. 5 (1954) pp. 808-812.

UNIVERSITY OF WASHINGTON 